\title{
Pierre-Louis Ginguené: la storia, la letteratura, la storia della letteratura
}

\section{Sergio Zoppi}

\section{Q OpenEdition \\ 1 Journals}

\section{Edizione digitale}

URL: http://journals.openedition.org/studifrancesi/7801

DOI: 10.4000/studifrancesi.7801

ISSN: 2421-5856

\section{Editore}

Rosenberg \& Sellier

\section{Edizione cartacea}

Data di pubblicazione: 1 juillet 2009

Paginazione: 337-340

ISSN: 0039-2944

\section{Notizia bibliografica digitale}

Sergio Zoppi, «Pierre-Louis Ginguené: la storia, la letteratura, la storia della letteratura», Studi Francesi [Online], 158 (LIII | II) | 2009, online dal 30 novembre 2015, consultato il 09 janvier 2021. URL: http:// journals.openedition.org/studifrancesi/7801 ; DOI: https://doi.org/10.4000/studifrancesi.7801

\section{(c)}

Studi Francesi è distribuita con Licenza Creative Commons Attribuzione - Non commerciale - Non opere derivate 4.0 Internazionale. 


\section{Pierre-Louis Ginguené: la storia, la letteratura, la storia della letteratura}

I rapporti culturali tra Francia e Italia tra Sette e Ottocento, in quell'epoca che vide l'Illuminismo orientarsi verso i filoni romantici delle letterature nazionali, sono egregiamente rappresentati dalla figura e dall'opera di Pierre-Louis Ginguené. Alla sua ricca personalità di giornalista e critico letterario e musicale, di storico e docente di letteratura, è doveroso riconoscere un importante ruolo nel dialogo tra i due paesi. Di là delle Alpi, erano gli anni dell'apogeo del concetto di nazione e di Stato, si imponeva il primato della Francia, della sua cultura e della sua lingua; sul nostro versante, invece, emergevano con sempre maggior vigore e chiarezza le esigenze di libertà, indipendenza e unità sul piano politico. In quelle istanze trovava espressione nei letterati la necessità di rintracciare e testimoniare le radici culturali italiane, e nel contempo un'attenzione via via più forte cresceva nei confronti della lingua italiana e della sua difesa anche come fattore unitario. Tale presa in carico di una lingua e di una cultura comuni alimentava quella primitiva, simbolica, unificazione del Paese, sulla quale poi si cementò lo spirito dell'età risorgimentale. Pochi anni dopo la scomparsa di Ginguené scoppiarono i primi moti insurrezionali per l'indipendenza e una seconda ondata di patrioti - dopo la generazione che si era distinta nelle Repubbliche del 1798-'99 - prese la strada dell'esilio o pagò, con la morte o il carcere, l'impegno per la liberazione dall'ennesimo dominatore, l'Impero Asburgico.

Originario di Rennes, Ginguené fu tra i tanti giovani aspiranti letterati che, nella seconda metà del Settecento, abbandonavano la provincia natale per cercar fortuna e maggiori stimoli culturali nella capitale. Intellettuale eclettico, operò attivamente e in svariati ambiti sulla scena culturale e anche politica francese sin dal 1772, anno del suo trasferimento a Parigi, e poi in particolare sotto il Direttorio e il Consolato. Negli anni '80 si distinse come poeta 'alla moda' e come critico musicale, e, nella querelle tra Gluck e Piccinni che tanto infiammò teatri e salotti parigini, si schierò con decisione tra i fautori del compositore barese. Durante la Rivoluzione si avvicinò alla compagine girondina e nel 1794, come André Chénier, pagò con alcuni mesi di carcere la sua ostilità al regime robespierrista; dopo Termidoro, con l'instaurazione di un governo moderato, assolse la funzione di direttore dell'Istruzione Pubblica e venne poi eletto al Tribunat. Nel 1798 rappresentò la Francia a Torino in qualità di ambasciatore presso Carlo Emanuele IV, re di Sardegna, nei mesi che precedettero l'invasione del Piemonte e la sua annessione alla Repubblica francese. Rientrato in patria, partecipò attivamente ai lavori della Classe de Sciences Morales et Politiques dell'Institut de France, dove si riunivano filosofi, storici, archeologi e letterati che si ispiravano al pensiero dell'Idéologie. Dopo aver ricoperto la cattedra di Histoire littéraire moderne all'Athénée dal 1802 al 1807, si concentrò sulla stesura di una Histoire littéraire d'Italie che, nel disegno iniziale, avrebbe dovuto costituire la prima parte di una più ampia Histoire littéraire moderne da lungo tempo progettata.

Nell'esercizio delle sue funzioni dimostrò costante interesse per le vicende italiane, per quel paese che lo aveva affascinato sin dalla più giovane età, quando si era accostato alla lingua, alla letteratura e alla musica per mai più allontanarsene, al punto 
da diventarne un fine conoscitore e portavoce nella sua terra. Lo studio del passato - storico, artistico, musicale e letterario - dell'Italia fu da lui condotto parallelamente a un continuo e preciso aggiornamento sulle vicende contemporanee della penisola, dagli accadimenti politici alle discussioni sulla 'questione della lingua', dalle novità in campo musicale all'evoluzione delle lettere. Studioso della letteratura italiana, si impegnò a fondo a farla conoscere e apprezzare in Francia, e si distinse per l'approfondita conoscenza anche della lingua, di cui fu estimatore e strenuo difensore; aspetto, quest'ultimo, assai originale in anni in cui il francese dominava incontrastato nell'Europa della cultura e della diplomazia quale 'lingua franca', mentre in Italia si discuteva, con toni talora accesi, della politica linguistica da adottare dinanzi all'«infranciosimento» delle terre passate in mano a Bonaparte.

L'opera principale di Ginguené, l'Histoire littéraire d'Italie, è un importante riconoscimento dell'identità culturale della penisola e delle sue antiche radici. Di esse egli intendeva ripercorrere le origini e lo sviluppo, al fine di illustrarne la priorità cronologica rispetto alle altre culture nazionali, la cui attestazione era stata favorita dall'identificazione secolare tra nazione e stato politico. Di quell'Italia che tanto lo appassionava non ebbe modo di visitare però che Torino nella breve parentesi del 1798. Tuttavia, intensi erano gli scambi epistolari con tanti letterati della penisola e, nel contempo, si annoveravano numerosi, fra gli ospiti della sua dimora parigina - oppure nella residenza estiva di Saint-Prix - gli scrittori e gli intellettuali italiani di passaggio a Parigi o, più di frequente, che a Parigi avevano trovato rifugio dopo la caduta delle Repubbliche Cisalpina e Napoletana. Ecco, dunque, Ginguené stringere amicizia e avviare attive collaborazioni con Carlo Botta, Ippolito Pindemonte, Francesco Salfi, e tanti altri ancora, che spesso aiutò a inserirsi nel mondo delle lettere e del giornalismo della capitale. Grazie a essi, sempre forieri di informazioni e di libri appena usciti dai torchi di tipografi italiani, Ginguené, pur non allontanandosi da Parigi, poteva aggiornarsi puntualmente sulle novità letterarie italiane, le quali costituivano l'oggetto di molte sue recensioni per «La Décade philosophique, littéraire et politique» (poi «La Revue philosophique, littéraire et politique») e, in seguito, per il «Mercure de France».

Troppo spesso adombrata da illustri suoi contemporanei, la figura del Ginguené ha destato l'attenzione di Paul Hazard, che per primo, all'inizio del Novecento, ne intuì il ruolo di passeur tra cultura italiana e francese tra XVIII e XIX secolo (cfr. La Révolution française et les lettres italiennes 1789-1815, Parigi, Hachette, 1910). Per trovare il primo studio d'insieme che tenga conto dell'uomo e dell'opera, con un'attenzione particolare all'Histoire littéraire d'Italie, occorre attendere il saggio di Marisa Zini, Il Ginguené e la letteratura italiana, pubblicato nel 1930 sul «Giornale storico della letteratura italiana» (vol. XCV, fasc. 285, 1930, pp. 209-242 e vol. XCVI, fasc. 286-287, 1930, pp. 1-38), rielaborazione della sua tesi di laurea discussa all'Università di Torino. L'attenzione verso Ginguené riaffiorò poi verso la fine degli anni Sessanta, con alcuni nostri contributi orientati a una ricostruzione della figura del giornalista e del critico letterario, autore di significativi interventi sulla stampa periodica riguardanti la cultura italiana (P.-L. Ginguené journaliste et critique littéraire. Textes choisis avec une introduction et des notes par S. Zoppi, Torino, Giappichelli, 1968). La ripresa degli studi su questo autore fu anche l'occasione per dare inizio alle ricerche nei suoi archivi, proseguite poi da Cristina Trinchero, che nel 2004 ha pubblicato una monografia sul Ginguené (Pierre-Louis Ginguené 1748-1816 e l'identità nazionale italiana nel contesto culturale europeo, prefazione di Sergio Zoppi, Roma, Bulzoni, 2004). Nel volume, gli interventi di Ginguené storico e critico della letteratura sono presi in esame nel più ampio discorso proposto dall'autore, quel fil rouge che, coerentemente con gli interessi e le intuizioni degli Idéologues, si rivela un'assai moderna riflessione sull'importanza della conoscenza e della valorizzazione degli interscambi culturali 
fra paesi e civiltà anche lontani. Nel 1992, nel quadro delle iniziative organizzate attorno al bicentenario della Rivoluzione, un convegno era stato dedicato a Ginguené nella sua città natale da Edouard Guitton, al quale va riconosciuto il merito di aver incoraggiato la ripresa degli studi attorno a questa figura, aprendo un ampio ventaglio di prospettive di ricerca (Ginguené 1748-1816, Idéologue et médiateur. Colloque international, Bibliothèque Municipale de Rennes, 2-4 avril 1992. Textes réunis par É. Guitton, Rennes, Presses Universitaires de Rennes, 1995). Ora, con il bel libro di Paolo Grossi, si aggiunge un nuovo e importante tassello nella storia degli studi su questo autore.

Paolo Grossi sembra aver raccolto la proposta avanzata da Roland Mortier nel convegno di Rennes («Reste à faire la biographie de Ginguené»), perché nel suo volume risalta un cospicuo e documentatissimo quadro de l'bomme et l'auvre, esito di scrupolose ricerche su libri e in archivi al fine di colmare finalmente un vuoto che troppo pesava nella bibliografia. Nella prima parte di Pierre-Louis Ginguené, historien de la littérature italienne (Bern, Peter Lang, 2006), infatti, l'itinerario biografico è seguito cronologicamente e suddiviso in momenti specifici: la giovinezza e la formazione a Rennes, il trasferimento a Parigi dove acquistò una discreta fama come poeta e librettista, l'attiva collaborazione a importanti periodici, gli incarichi politici e amministrativi con il Direttorio e il Consolato, la cattedra all'Athénée e, infine, gli ultimi anni, dove l'impegnativa stesura della poderosa Histoire littéraire d'Italie si alternava a momenti di evasione nella sempre amata poesia.

Nelle parti II, III e IV del volume, Paolo Grossi concentra poi l'attenzione su Ginguené italianista, soffermandosi sugli anni in cui fu docente all'Athénée, dove sperimentò un metodo di insegnamento della letteratura italiana che avrebbe ispirato l'Histoire littéraire d'Italie. In particolare, nella parte II, Grossi ricorda la genesi del progetto per una storia letteraria moderna dai primi abbozzi presenti sulle pagine de «La Décade» e del «Mercure», sottolineandone la novità per l'epoca. A queste premesse segue l'analisi della Préface all'Histoire, dove Ginguené, approfondendo quanto anticipato anni prima, spiega con precisione le finalità del suo lavoro e, soprattutto, il suo metodo di studio della letteratura, basato su alcune linee portanti: il concetto di letteratura nazionale, la prospettiva cronologica, la collocazione di autori e opere nel loro contesto storico-politico-sociale, in quello che egli amava indicare con l'espressione «cadre». A questa prefazione, che descrive l'impostazione dei volumi dell'Histoire littéraire d'Italie e che nel contempo costituisce un vero e proprio saggio atto a illustrare un nuovo, moderno, per l'epoca, metodo di studio della letteratura, Paolo Grossi dedica il dovuto spazio: quella del Ginguené si presenta come una storia letteraria che, pur facendo tesoro dei capisaldi della storiografia letteraria del XVIII secolo - i volumi di Tiraboschi, Bettinelli, Denina, ecc. - ebbe il pregio di svincolarsi dal gusto per l'erudizione e dalla compilazione acritica a vantaggio di una riflessione sul percorso dei generi letterari, delle poetiche e dell'estetica attraverso i secoli e le società, e dei singoli autori all'interno della loro vita e opera, realizzando quella che il Salfi definì, recensendola, come una storia letteraria «philosophique». Non ultimo, Paolo Grossi sottolinea un'altra importante caratteristica di Ginguené: l'avvicinamento del lettore o del discente ai testi, l'analisi delle opere letterarie, l'esaltazione del piacere della lettura, di quell'atto che Gustave Lanson avrebbe definito la «degustazione» della letteratura.

La parte III del volume è dedicata all'esame dei tomi che compongono l'Histoire, dei quali vengono ricordati l'iter di composizione e di pubblicazione, il piano di lavoro, l'impostazione, i contenuti e i criteri di periodizzamento della letteratura italiana. Nella parte IV Paolo Grossi riassume gli aspetti salienti dello 'storicismo' di Ginguené, che definisce autore di «une histoire "philosophique" et "généalogique"», e si concentra sulle due sezioni più ampie dell'Histoire littéraire d'Italie, dedicate 
rispettivamente alla Divina Commedia e alla Gerusalemme liberata quali esempi della critica letteraria di Ginguené, in bilico tra intuizioni moderne e formazione di impostazione classica.

Il volume si chiude con una congrua appendice di carattere documentaristico, dove Grossi ha scelto di elencare 'tutti' gli scritti di Ginguené, anche articoli e recensioni pubblicati su periodici (perlomeno quegli articoli sinora individuati e individuabili), poi le voci per la Biographie Universelle Michaud, i titoli delle poesie pubblicate su «L'Almanach des Muses» e, infine, i programmi dei corsi tenuti all'Athénée. Segue una selezione di altri documenti con i quali lo studioso sembra aver voluto dare un piccolo saggio antologico di alcuni aspetti della figura del Ginguené: troviamo il toccante poema Convalescence, che si chiude con l'epitaffio voluto da Ginguené per la sua tomba, e alcune annotazioni di Jean-Baptiste Say inerenti la propria collaborazione a «La Décade», periodico di cui, lo ricordiamo, Ginguené era stato uno dei fondatori e il coordinatore, avvalendosi soprattutto della collaborazione di Amaury Duval. Con la bibliografia finale, molto articolata nella presentazione, Paolo Grossi si propone di riunire tutto lo stato dell'arte su Ginguené e sul suo tempo, stilando una vera e propria rassegna bibliografica aggiornata di indubbia utilità tanto per chi si accinge ad affrontare lo studio di questo autore quanto per chi necessiti di disporre delle indicazioni bibliografiche di riferimento essenziali per la storia della storiografia letteraria e, più in generale, per il contesto storico-culturale francese tra Sette e Ottocento. Risultato di ricerche e di letture attente, l'appendice e la bibliografia obbediscono al progetto che sottende tutto il lavoro del Grossi e che affiora di tanto in tanto: la volontà di realizzare una sintesi esauriente, riordinando e arricchendo gli studi già esistenti, di cui egli fa tesoro, ed espurgandoli, tramite una diligente verifica di dati e di fonti di prima mano, dalle imprecisioni che purtroppo spesso si sono accumulate e tramandate nel tempo.

L'impostazione, la chiarezza, le minute ricostruzioni storiche e il controllo meticoloso delle fonti attestano la serietà del lavoro, le doti di ricerca e la preparazione di Paolo Grossi. Pierre-Louis Ginguené, historien de la littérature italienne è dunque un contributo di sicuro interesse e di alto valore scientifico per la conoscenza e per una meritata rivalutazione di un autore di rilievo nel panorama generale delle relazioni culturali tra Francia e Italia tra Sette e Ottocento, e nel contesto specifico degli studi sulla storia della storiografia e della critica letteraria. Ne consegue che questo volume costituirà un punto di riferimento insostituibile per nuovi percorsi di ricerca, tanto attorno a Ginguené quanto sulla vita letteraria e gli scambi culturali tra Francia e Italia nel periodo in cui si colloca il suo operato. 